\title{
Téoros
}

Revue de recherche en tourisme

\section{Tourisme et patrimoine}

\section{Line Ouellet}

Volume 7, numéro 1, mars 1988

Cultures régionales et tourisme

URI : https://id.erudit.org/iderudit/1080426ar

DOI : https://doi.org/10.7202/1080426ar

Aller au sommaire du numéro

\section{Éditeur(s)}

Université du Québec à Montréal

\section{ISSN}

0712-8657 (imprimé)

1923-2705 (numérique)

Découvrir la revue

Citer cet article

Ouellet, L. (1988). Tourisme et patrimoine. Téoros, 7(1), 14-16.

https://doi.org/10.7202/1080426ar d'utilisation que vous pouvez consulter en ligne.

https://apropos.erudit.org/fr/usagers/politique-dutilisation/ 


\section{Tourisme}

et
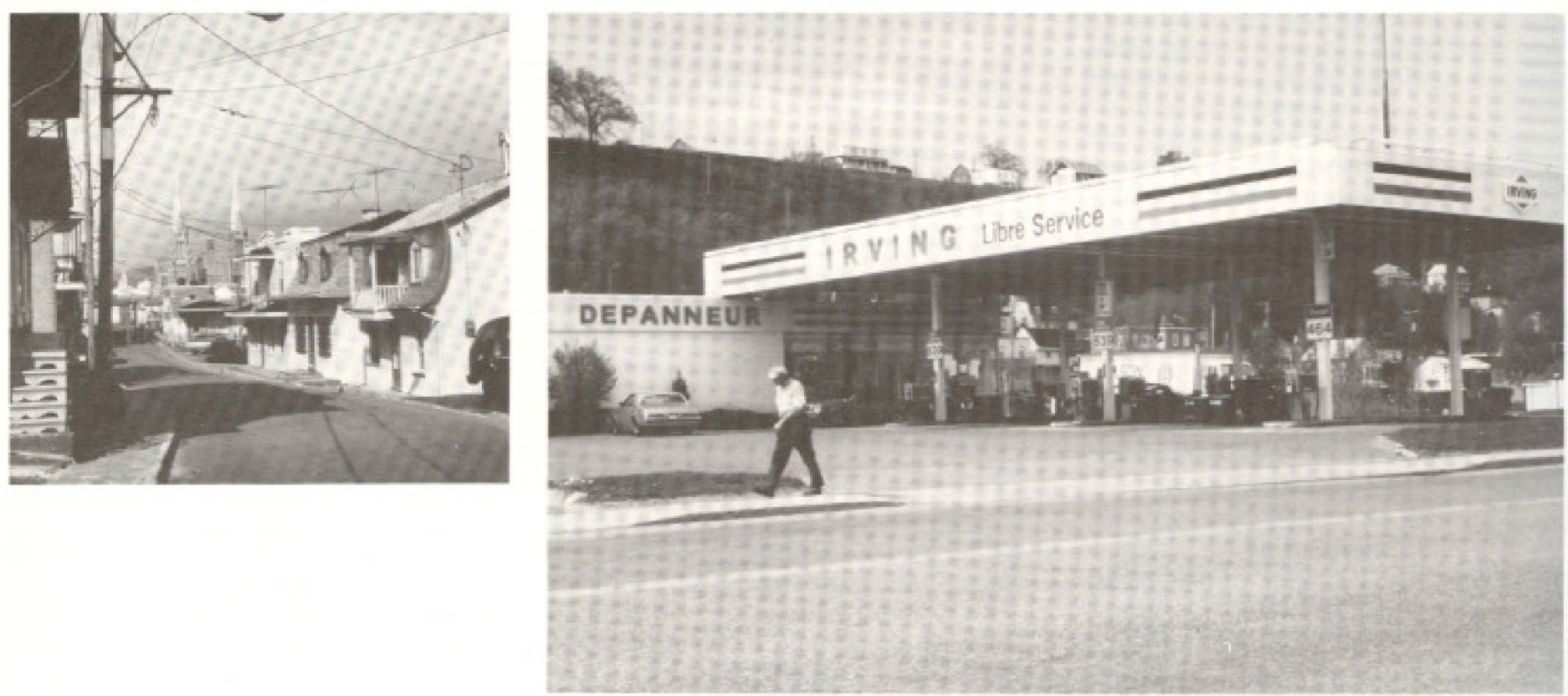

Le village avec sa succession de petites maisons, son tissu dense, et son dedale de fils électriques. A remarquer le peu d'espace pour les piétons. Tout a été empiété pour faire place à I'auto. (photo 1 à gauche)

Le "dêpanneur" Irving jumele a une marquise gigantesque qui pourrait protéger les clients même si ses proportions étaient réduites de moitié. Cette masse qui se découpe sur les verts tendres de la côte Sainte-Anne apparaît d'un ridicule encore plus achevé qu'en milieu urbain. (photo 2 à droite)
"Madame Ouellet est coordonnatrice drexposition au Musde de la civilisation, Oudbec; elle etait Directrice et rédactrice en chef de la recue Continuito su moment de la presentation de ce texte.
Le patrimoine est ici défini comme un ensemble dynamique d'idées, d'images. d'objets qui encore aujourd'hui interpellent les sens, stimulent l'esprit, animent l'espace. Si la culture comprend l'ensemble des modes de fonctionnement et de création d'une société, il va sans dire que l'héritage des siècles en constitue le fondement.

Nous nous attarderons particulièrement au paysage bâti au sens large du terme, incluant l'aménagement et l'environnement; au décor, en quelque sorte, pour reprendre le vocabulaire associé au jeu. Nous démontrerons d'abord que, depuis les années soixante surtout, le développement touristique au Québec s'est fait au détriment des caractères spécifiques du paysage. Sainte-Anne-deBeaupré servira fort bien notre propos.

Toutefois, à un tel constat, on doit juxtaposer une réflexion sur l'évolution des exigences de la clientẻle touristique et sur la vision récente du patrimoine québécois. Nous proposerons finalement une approche du développement touristique qui s'harmonise avec la mise en valeur du patrimoine en nous appuyant sur quelques projets réalises (ou en voie de réalisation) en Beauce.

\section{Le cas de}

\section{Sainte-Anne-de-Beaupré}

A l'image des autres villages de la cóte de Beaupré, le coeur traditionnel de Sainte-
Anne est marqué par une densification des habitations et la présence des institutions municipales et religieuses, qui s'élèvent de part et d'autre du chemin Royal, s'accrochant parfois à la falaise qu'il borde (voir photo 1).

Cette contrainte physique limitait, il va sans dire, le développement du noyau central du village soumis à d'importantes pressions touristiques, en particulier du tourisme religieux.

Le pôle de développement s'est done déplacé naturellement, comme pour tant d'autres villes et villages, "près de la grand route". Longeant le fleuve, le boulevard Sainte-Anne est parallèle à l'avenue Royale et séparé d'elle par quelques centaines de metres occupés entre autres par la voie ferrée. Deux atouts: proximité du fleuve, possibilité de maintenir un lien avec le coeur ancien situć à distance raisonnable malgré l'obstacle de la voie ferrée. Le résultats: tous les motels et autres services tournent le dos au fleuve et sont entoures de bitume; aucun lien $n$ "existe entre le coeur traditionnel du village et le coeur commercial.

De plus, l'architecture des bătiments commerciaux, en particulier ceux postérieurs aux années cinquante, est d'une banalité désarmante et ne tient pas compte (volumétrie, choix de matériaux, de couleurs), des caractéristiques du site et de l'architecture environnante (voir photo-2); celle-ci. 


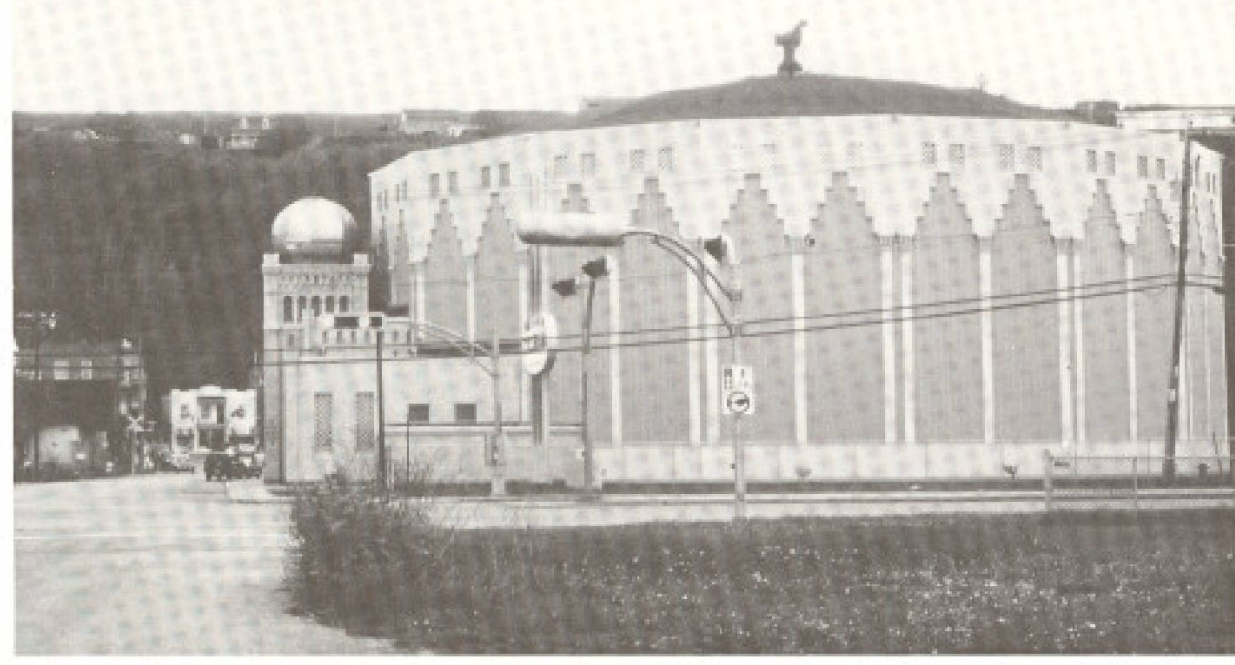

Les peintures murales de I'intérieur du cycloramátaient au départ des oeuvres fort intéressantes qui, après de nombreuses restaurations "peu respectueuses", sont devenues méconnaissables. (photo 3)

rappelons-le, est dominée par l'imposante stature de la basilique dessince par l'architecte français Maxime Roisin en collaboration avec Louis-Napolcon Audet et érigée sur une periode de plus de trente ans.

Sur le boulevard Sainte-Anne, les hơtels récents $s^{t} a l i g n e n t$ perpendiculairement au fleuve avec une dominante de couleur orange particulièrement choquante dans ce paysage domine par les bleus et les werts. C'est la premiere image qui $s^{7}$ offre aux visiteur's a l'entrée de Sainte-Anne sur le boulevard.

Aussi, dans le cas de Sainte-Anne, comme dans bien d'autres cas au Québec, le développement touristique et plus généralement immobilier, s'est fait à la piece avec le profit pour seule règle. Mais voilă, les arnées cinquante sont bien loin, les Québécois n'en sont plus à leur premier woyage et leurs exigences ont change. L'augmentation et la diversification de la clientele touristique exigent maintenant des promoteurs du tourisme québécois un raffinement de leur's produits.

\section{Fréquentation touristique et spécificité culturelle}

En effet, des études françaises prouvent que dans le domaine culturel. les touristes en vacances ont des pratiques différentes de celles des autres temps de l'année. Généralement, les pratiques culturelles s'intensifient au cours des yacances. Si on se represente la clientele touristique sous forme d'une pyramide, on retrouverait à la pointe, selon des souroes françaises, une portion de touristes $(29 \%, 1974)$ qui, vacances ou non, connaissent et apprécient l'art, la littérature ou la science.

Ce sont les touristes "cultives" au sens restreint de la culture classique. La majorité est assez scolarisee et fait partie des classes moyennes et superieures. (Il s"agit ici d'un portrait robot). Une autre portion est qualifiée de culture populaire $(27 \%)$, c'est-à-dire amateurs de fêtes foraines, de variétés: ils pratiquent des loisirs actifs tels le jardinage et le bricolage. Ses tenants sont moins scolarises et se recrutent plus largement chez les ouvriers et les agriculteurs mais aussi, en partie, dans les classes supérieures. A la base de la pyramide avec $44 \%$, les "indifferents" à la culture sortent peu et sont branchés sur les programmes populaires de télévision. $\mathrm{Co}$ serait cette clientèle qui est la plus susceptible-de modifier ses habitudes lors d'un voyage d'agrément et de tâter certaines pratiques culturelles.

Revenons a Sainte-Anne: Quel type de clientèle a-t-on le plus de chance de retrouver a la basilique? On peut supposer qu'une forte proportion de ces touristes ont des pratiques culturelles peu nombreuses en dehors des vacances. Et pourtant, les voila sur un site très signifiant sur le plan culturel. En effet, la basilique Sainte-Anne combine une séric d'avantages qui font d'elle un haut lieu touristique de la région (avec les chutes Montmorency et l'île d'Orléans).

La basilique a acquis une notoriéte historique d'abord sur le plan religieux avec ses nombreux miracles. Elle est ainsi devenue le Lourdes du Québec. Notoriété à laquelle s'ajoute le caractere monumental de la cathédrale. Aussi notoire ou monumentale qu'elle soit, elle ne profiterait toutefois pas d'un tel flot touristique si elle n'était pas situé à moins de trente minutes de la vieille capitale, et si elle n'était pas extrêmement visible et facile d'access de l'autoroute: accessibilité, notorićté et monumentalisme d'un site sont souvent plus determinants de sa fréquentation que sa qualite intrinsèque.

Parmi les composantes de qualité à SainteAnne, mentionnons: le Musé de l'Historial

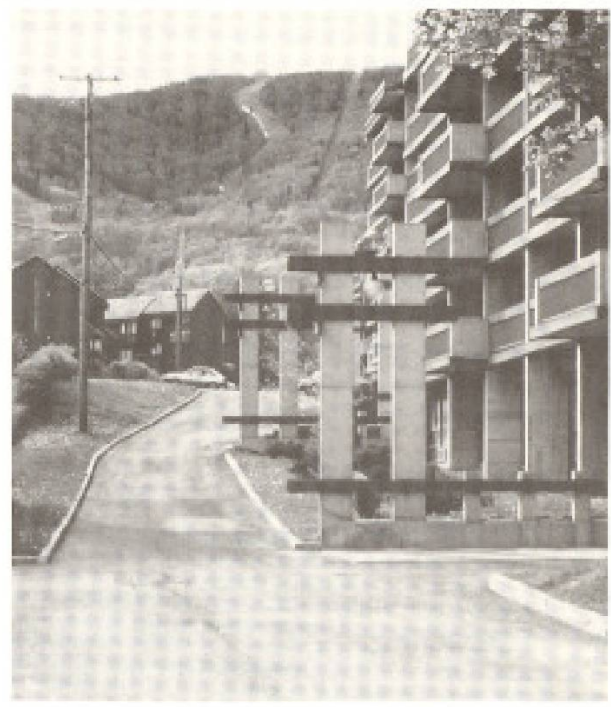

Le seul espace oũ le hasard plutôt qu'une planification consciente a rendu possible I'existence d'une rue à áchelle humaine qui encadre une perspective sur la montagne. (photo 4) qui possède des collections d'art sacré prestigieuses; y est aussi conservée la maquette de la basilique, typique des grandes maquettes de l'architecture beaux-arts; le chemin de croix dont les statues proviennent, comme pour bien d'autres sites religieux au Québec, de l'atelier de Vaucouleurs en France; la petite chapelle reconstruite à partir de vestiges du XVIIe siècle dont les proportions sont exquises et à quelques pas la Santa Scala, un coin d'Italie sur la côte de Beaupré; le cyclorama dont l'architecture a été banalisée par le recouvrement d'aluminium (voir photo 3 ).

Malgré ce potentiel indéniable, on offre trop souvent aux nombreux touristes une mise en capsule de miracles afin de stimuler leur sens religieux. Cette réduction de l'information et sa commercialisation mettent en péril, en la banalisant, la ressource touristique mẻme.

\section{Sainte-Anne et Mont Sainte-Anne}

Ainsi, Sainte-Anne qui est le plus gros village à proximité du vaste complexe touristique du Mont Sainte-Anne, profite d'un flot de touristes et villégiateurs dont une portion saurait apprécier le cachet de son village, les qualités architecturales de sa basilique et la beauté naturelle du fleuve.

Au lieu de cela. le complexe du Mont SainteAnne se referme sur lui-meme en offrant tous les services: hébergement, restaurants, bar, ski, golf, tennis, piscine... Même si l'expsrience française dans les Alpes a prouvé depuis dix ans la monstruosité de ces vastes constructions nouvelles aux textures brutes. et aux gabarits qui brisent l'echelle humaine, nous persistons ici à faire de même et détruisons du mème coup la subtile harmonie entre la nature et la culture d'une région (voir photos 4 et 5 ). Soulignons toutefois que certaines auberges démontrent une volonté d'offrir' un produit plus respectueux de la spécificité régionale. 
Cette tendance à la banalisation et à la réduction des ressources touristiques parmi lesquelles figurent au premier plan le patrimoine architectural des Québécois n'existe pas qu'à Sainte-Anne-de-Beauprć. C'est un phénomène que l'on constate dans de nombreuses régions du Québec. Et du point de vue de la conservation du patrimoine architectural, on peut presqu'avancer que moins la région est développée, mieux elle cst conservée! C'est le cas de Lanaudière que Monsieur Morissonneau a mis en lumière avec son guide.

\section{Développement et patrimoine}

Le patrimoine architectural québécois n'est pas composé que d'édifices que l'on peut qualifier de "monuments" au sens de la loi des biens culturels. Outre les sites et monuments classés, il existe au Québec une architecture domestique, commerciale, industrielle et institutionnelle qui constituc une vaste mosaique dont la valeur d'ensemble dépasse la somme des valeurs individuelles. Cette constatation est à la base d'une approche en architecture en urbanisme que l'on nomme "contextualisme". Ainsi, doiton non seulement conserver des monuments et sites exemplaires mais permettre que l'ensemble du patrimoine bâti évolue par les constructions nouvelles ou par la restauration ou le recyclage. Cette évolution doit se faire dans le respect des caractéristiques fondamentales de chacun des bâtiments sur lesquels on intervient et des sites sur lesquels on construit (voir photo 6).

Cette approche globale, qui relie la conservation et la création et perçoit le patrimoine non seulement dans ses composantes exceptionnelles mais également dans son ensemble en évolution, $n$ 'est pas encore largement répandue au Québec. Elle apparaît de façon ponctuelle, dans le cas de certains projets. Il va sans dire que cette approche, puisqu'elle encourage la sensibilité aux caractéristiques d'un édifice ou d'un ensemble, contribue à la mise en valeur et à l'évolution harmonieuse du patrimoine architectural qui est une composante cssentielle de la spécificité culturelle du Québec. La relation entre patrimoine architectural et mise en valeur touristique doit être vu dans ces mểmes termes.

Ainsi, tout développement à des fins touristiques ou non, devrait tenir compte des possibilités de réutilisation des nombreux bâtiments historiques, classés ou non, et de critères de design en relation avec l'environnement du site.

Un programme fort ambitieux certes, qui appelle une vaste collaboration entre les municipalités qui définissent actuellement leur plan d'urbanisme, les promoteurs de l'industrie touristique qui doivent maintenant faire face à une demande plus exigeante, les professionnels de l'environnement bâti (architectes, urbanistes, historiens de l'architecture) qui sont de plus en plus sensibles à la nécessité de protéger notre héritage bâti et de créer l'héritage de l'avenir.

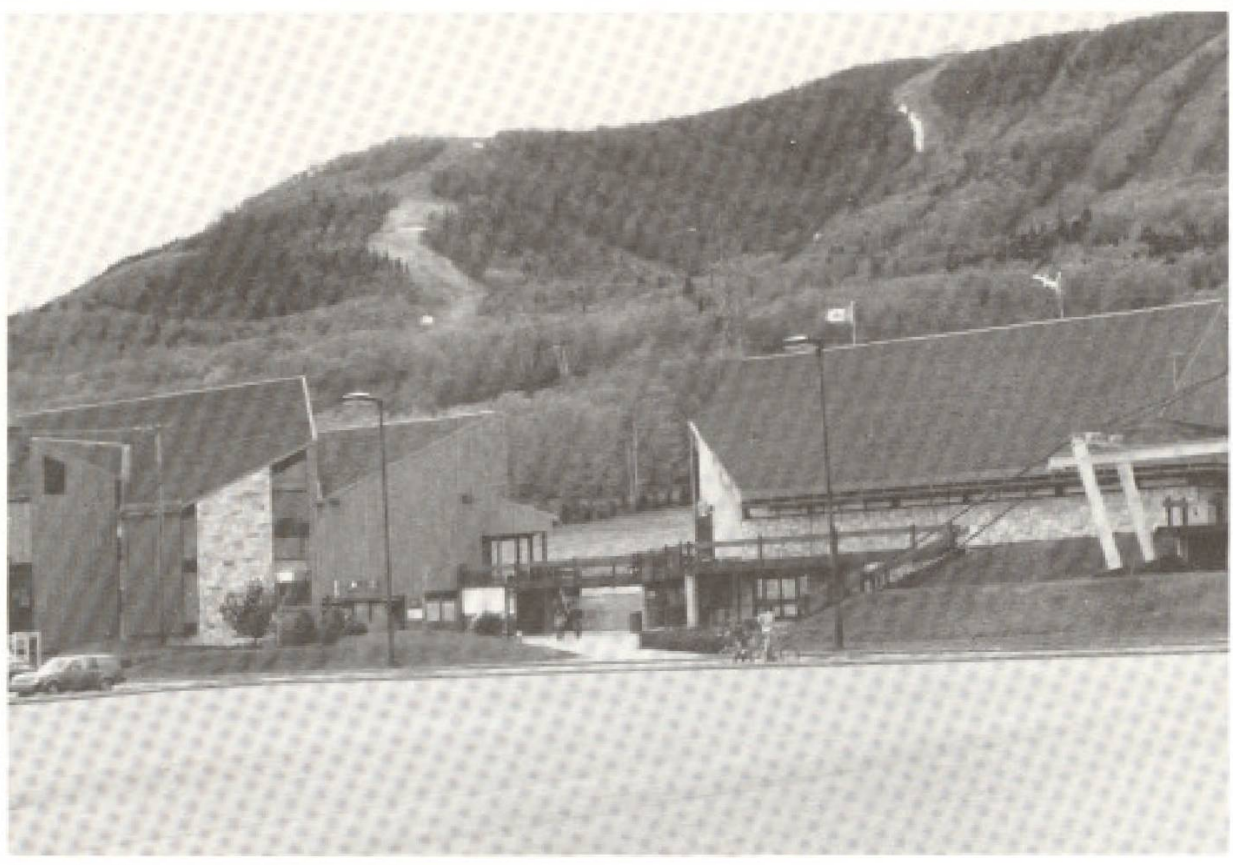

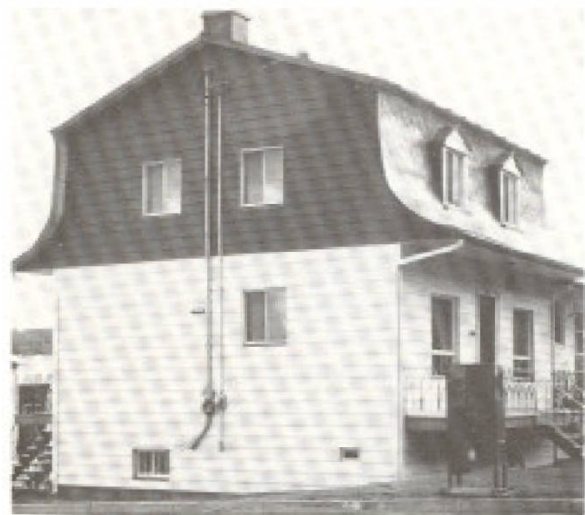

\section{L'exemple de la Beauce}

L'exemple du site institutionnel de Saint= Joseph-de-Beauce, premier site du genre classé au Québec (1985), est très pertinent. Il regroupe cinq bătiments: l'église, le presbytère, le couvent, l'orphelinat et l'école. Le couvent loge maintenant le Musée Marius-Barbeau et de nombreux organismes culturels dont la Société du patrimoine des Beaucerons. L'orphelinat abrite des services communautaires. Les autres bătiments ont conservé leur fonction d'origine. Un plan directeur d'aménagement a ćté commandé à la firme Pluram afin de redonner à cet ensemble une cohérence que les nombreux espaces de stationnement rendaient moins visible.

La Société du patrimoine des Beaucerons, en collaboration avec le ministère des Affaires culturelles et la Fondation Robert-Cliche, a produit un dépliant riche en informations sur I'histoire de la ville et l'arehitecture des bâtiments du site. En collaboration toujours avec le ministère et cette fois avec les municipalités régionales de comté, la Société a également conçu un itinéraire patrimonial de la Beauce qui témoigné d'une volonté ferme de faire connaître et reconnaitre l'héritage beau-
Le chalet à la base de la montagne. Une série de volumes et de textures sans grande cohé rencè. Malgré I'utilisation de matériaux nobles tels le bois et la pierre, le traitement est tel que c'est le bardeau $d^{\prime}$ asphalte de la toiture qui domine... (photo 5 en haut)

Une mansarde qui a subi un traitement à l'aluminium; elle $\gamma$ a perdu ses chambranles et menaux de fenêtres ainsi que les arêtes marquant les coins de la maison. (photo 6 en bas).

ceron aux visiteurs et aux visités.

Cet itineraire indique les monuments et sites classés ainsi que de nombreux autres "biens culturels" de la Beauce. Il exploite les points de vue et perspectives les plus significatifs en mettant I"accent sur des ensembles. Voilà le type d'instrument dont les régions du Québec auraient besoin pour faire connaitre et reconnaittre leur héritage architectural aux visiteurs et visités.

\section{Conclusion}

De nombreuses municipalités régionales de comté présentent dans leur schéma d'aménagement un volet tourisme et patrimoine en identifiant les concentrations ou les bâtiments d'intérêt. Il s'agit là̀ d'un premier pas. Les municipalités doivent maintenant produire leur plan particulier d'urbanisme qui permettront de baliser les types de développement immobilier. Cette vaste opération de planification doit tenir compte de la conservation et du potentiel des bâtiments existants et de la nécessaire intégration des nouveaux bâtiments dans leur environnement. Sans quoi, nous n'aurons que quelques monuments exceptionnels "momifiess" à offrir à nos visiteurs et, bien sûr, aux générations futures. $\boldsymbol{f}$ 